\title{
Psychological Correlates of Handedness and Corpus Callosum Asymmetry in Autism: The left Hemisphere Dysfunction Theory Revisited
}

\author{
Dorothea L. Floris • Lindsay R. Chura • \\ Rosemary J. Holt • John Suckling • Edward T. Bullmore • \\ Simon Baron-Cohen • Michael D. Spencer
}

Published online: 23 November 2012

(c) Springer Science+Business Media New York 2012

\begin{abstract}
Rightward cerebral lateralization has been suggested to be involved in the neuropathology of autism spectrum conditions. We investigated functional and neuroanatomical asymmetry, in terms of handedness and corpus callosum measurements in male adolescents with autism, their unaffected siblings and controls, and their associations with executive dysfunction and symptom severity. Adolescents with autism did not differ from controls in functional asymmetry, but neuroanatomically showed the expected pattern of stronger rightward lateralization in the posterior and anterior midbody based on their hand-preference. Measures of symptom severity were related to rightward asymmetry in three subregions (splenium, posterior midbody and rostral body). We found the opposite pattern for the isthmus and rostrum with better cognitive and less severe clinical scores associated with rightward lateralization.
\end{abstract}

Keywords Autism - Corpus callosum - Handedness · Asymmetry $\cdot$ Lateralization · Broader autism phenotype

In this paper we have adopted the terminology ASC in preference to "Autism Spectrum Disorders (ASD)", as we consider this terminology to be less burdensome to individuals and families affected. To avoid confusion, however, we would point out that we are using ASC to refer to the same conditions as ASD.

D. L. Floris $(\bowtie) \cdot$ L. R. Chura · R. J. Holt · S. Baron-Cohen · M. D. Spencer

Department of Psychiatry, Autism Research Centre, University of Cambridge, Cambridge, UK

e-mail: df312@medschl.cam.ac.uk

J. Suckling · E. T. Bullmore

Department of Psychiatry, Herchel Smith Building for Brain and Mind Sciences, University of Cambridge, Cambridge Biomedical Campus, Cambridge, UK

\section{Introduction}

The neuroanatomical underpinnings of autism spectrum conditions (ASC) are still poorly understood, but theories surrounding atypical cerebral asymmetry as one possible factor influencing the condition have received considerable attention. Many people with ASC show a pattern of deficits in skills ascribed to the left hemisphere, such as language, communication and symbol use, whilst appearing relatively unimpaired in right hemisphere functions such as visuospatial abilities (Blackstock 1978; Fein et al. 1984; Prior and Bradshaw 1979). These observations have given rise to the notion that the condition might be related to atypical cerebral organization, with the left hemisphere being most affected.

\section{Evidence from Neuroimaging Studies}

Further evidence for this 'left hemisphere dysfunction' theory stems from neuroimaging studies showing a reversal of asymmetry in language-related regions such as the inferior lateral frontal cortex (De Fosse et al. 2004; Herbert et al. 2002), the parieto-occipital region near the posterior language zone (Hier et al. 1979) and the planum temporale (Rojas et al. 2002, 2005). These alterations of typically occurring asymmetries are corroborated by findings based on single photon emission computed tomography (Chiron et al. 1995; Ohnishi et al. 2000), and positron emission tomography (Muller et al. 1999) showing atypical or reversed cerebral blood flow in frontal language regions. Other brain regions have been implicated, too. Computerized tomography (CT) scanning reveals increased cerebral blood flow in the right temporal and right parietal lobes (Hashimoto et al. 2000). Siegel et al. (1992) found leftward reductions in glucose metabolism in the posterior putamen. 
Also, the amygdala was shown to display reduced leftward volume in individuals with autism (Rojas et al. 2004).

Evidence from Studies on Functional Asymmetry

Another source of evidence for left hemisphere dysfunction is research into functional asymmetry. On dichotic listening tasks, children with ASC do not show the usual right ear advantage for speech processing (Prior and Bradshaw 1979). They tend to prefer to use their left ear when listening to verbal and musical stimuli, compared to typically developing children who prefer to use their left ear for musical and their right ear for verbal stimuli (Blackstock 1978). Studies of handedness, as another marker of lateral preference, support these findings. It is well documented that there are elevated rates of left-handedness in ASC (Colby and Parkison 1977; Gillberg 1983; Hauser et al. 1975; McCann 1981; McManus et al. 1992; Soper et al. 1986; Tsai 1983). However, many studies also report a high incidence of ambiguous or inconsistent lateral preference (Bryson 1990; Cornish and McManus 1996; Fein et al. 1984; Soper et al. 1986). Thus, 30-40 \% of children with ASC display inconsistent hand preference, whereas $15-20 \%$ exhibit established left-hand preference, which is almost twofold increase compared to the general population (Leboyer et al. 1988). Mixed-handed individuals with ASC score lower on cognitive tasks in comparison to individuals with ASC with consistent hand preference (Fein et al. 1984; Soper et al. 1986).

\section{Cerebral Connectivity and the Corpus Callosum}

There is a close link between cerebral lateralization and connectivity, with more strongly lateralized brains relying on less inter-hemispheric transfer. In particular, the corpus callosum has emerged as a target of research into cerebral connectivity owing to its associations with functional asymmetry and other structural brain asymmetries (Aboitiz et al. 1992; Clarke and Zaidel 1994; Dorion et al. 2000; Hopkins and Rilling 2000; Witelson and Goldsmith 1991). It is the main fibre tract mediating inter-hemispheric communication between (mostly) homologous cortical areas, which are critical for higher cognitive functions (Tomasch 1954). The corpus callosum is topographically organized such that different subsections project to different cortical regions (see Fig. 1). This distributional pattern of the inter-hemispheric fibres is of essential importance as regional characteristics shed light on the organization of associated cortical areas.

The majority of studies have focused on volumetric differences between the sexes and handedness groups (Allen et al. 1991; Tepest et al. 2010; Witelson 1989), or clinical subgroups (Foong et al. 2000; Hardan et al. 2000;

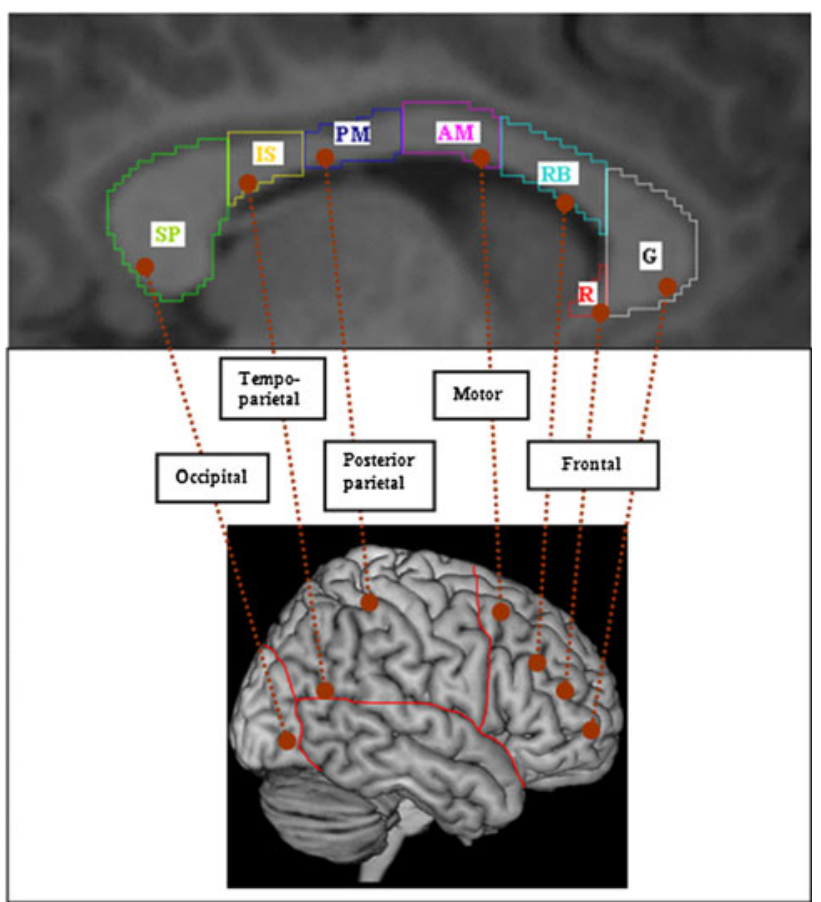

Fig. 1 Anatomical sub-regions of the corpus callosum and cortical regions it projects to according to Witelson (1989). Subdivisions of the corpus callosum in seven regions after tracing and parcellating in AnalyzeDirect, Inc., KS: $S P$ splenium (projects to occipital, inferior temporal cortex), IS isthmus (projects to superior temporal, posterior parietal cortex), $P M$ posterior midbody (projects to somatosensory, posterior, parietal cortex), $A M$ anterior midbody (projects to motor cortex), $R B$ rostral body (projects to premotor, supplementary motor cortex), $G$ genu (projects to prefrontal cortex), $R$ rostrum (projects to caudal/orbital prefrontal, inferior premotor cortex). Upper figure shows manually traced outline, projected for illustration onto a representative brain image in coronal section. Lower figure shows 3D rendered MNI template image in MRIcron

Hynd et al. 1991). One of the most reliable findings is that consistently right-handed men have a smaller corpus callosum than non-consistently right-handed men (Witelson 1985, 1989). With respect to ASC, overall size reductions have been reported (Brambilla et al. 2003; Hardan et al. 2000, 2009).

Considering its topography, one might assume that hemispheric lateralization might not only relate to callosal size but also co-occur with callosal asymmetries. As there is an inverse relationship between cortical asymmetry and corpus callosum volume (Galaburda et al. 1990), global callosal size reductions (in association with greater hemispheric and functional lateralization) may be mediated by regional right or leftward callosal reductions, depending on which hemisphere exhibits dominance. However, to date, only one study has tested whether there are structural asymmetries of the corpus callosum itself. Luders et al. (2006) were the first to suggest that the distribution of callosal fibres is influenced by structural differences in homologous cortical regions. They report stronger 
rightward lateralization in the anterior callosal section which projects to the motor cortices in right-handed males, concluding that stronger leftward lateralization of motor functions in right-handers reduces left commissural connections. Despite the obvious functional relevance of corpus callosum asymmetries, no other detailed study has been performed in either typical people or any clinical population.

Callosal size reductions in autism are in line with theories suggesting that ASC is a condition of neural underconnectivity and deficits result from reduced synchronization between different brain regions (Just et al. 2004). Also, Courchesne and Pierce (2005) and Belmonte et al. (2004) argue that underlying neural defects in ASC are due to reduced long distance connectivity between the frontal cortex and other systems, and to local over-connectivity in the frontal lobe. In fact, individuals with ASC display impairments in fronto-striatal higher-order functions in social, emotional and cognitive domains, such as social and flexible behaviour and neuropsychological performance. Accordingly, they have impaired performance on executive function tasks such as the Wisconsin Card Sorting test (WCST; Berg 1948), the Tower of Hanoi (Borys et al. 1982), and two subtests of the CANTAB involving frontal function-the Stockings of Cambridge and the Intradimensional/Extra-dimensional Shift task (Ozonoff et al. 2004). Even though factors related to these neuropsychological deficits remain unclear, it has been suggested that impairments in executive function might be caused by atypically rightward lateralized fronto-striatal network systems deficient in integrating information (Rinehart et al. 2001). Both neuroanatomical and functional connectivity alterations have thus been proposed to be core elements of the pathophysiology of ASC.

\section{The Present Study}

The purpose of the present study was to examine whether adolescents with ASC display both atypical functional asymmetry in handedness, and atypical neuroanatomical asymmetry in the corpus callosum. We expected individuals with ASC to show a leftward handedness shift and more pronounced rightward lateralization. A further aim was to explore whether atypical lateralization is related to social, cognitive and language functions, which are impaired in ASC and have been related to aberrant connectivity (Courchesne and Pierce 2005). Cognitive functions were assessed using IQ and neuropsychological performance on two tasks from the CANTAB (Robbins et al. 1998) measuring executive functioning. Clinical symptoms were assessed using the Autism Diagnostic Observation Schedule-Generic (Lord et al. 2000) and the Autism Diagnostic Interview-Revised (Lord et al. 1994).
Finally, as ASC is strongly genetic, with heritability being estimated around 60-90\% (Bailey et al. 1995; Folstein and Rutter 1977; Folstein and Rosen-Sheidley 2001; Ronald et al. 2006; Steffenburg et al. 1989; Sullivan et al. 2012), we compared people with ASC not only to controls, but also to their unaffected siblings, to shed light on a potential neuroanatomical endophenotype of autism. Initial functional MRI findings in this cohort have already been described (Spencer et al. 2011).

\section{Method}

Participants

Participants comprised 40 adolescents with high-functioning autism (HFA) or Asperger Syndrome, 40 unaffected, full biological siblings of those in the autism/Asperger group, and 40 typically developing controls. Further recruitment details of this cohort have been described previously (Spencer et al. 2011). All participants in the autism group were positive on both the ADOS-G (Lord et al. 2000) and ADI-R (Lord et al. 1994). All siblings and controls scored below threshold $(<15)$ on the Social Communication Questionnaire (SCQ; Berument et al. 1999). Based on self-reports on the Kiddie Schedule for Affective Disorders and Schizophrenia (K-SADS; PuigAntich et al. 1987) and parents' reports on a family history questionnaire, controls did have neither any psychiatric disorder nor any first- or second-degree family members with ASC. Subjects were excluded if they met any of following criteria: (1) a current IQ less than 70, as assessed by the Wechsler Abbreviated Scale of Intelligence (WASI; Wechsler 1999); (2) any current or previous use of psychotropic medication; (3) any psychiatric diagnosis (other than an ASC in the autism group); (4) any history of seizures, drug abuse, intracranial surgery or head injury.

In view of the well-characterized sexual dimorphism in brain structure (Giedd et al. 1997), for this study we focused only on the male sub-sample. This comprised 35 adolescents with autism ( $M$ age: 14.48 years; $S D$ : 1.74; range: $12.01-18.53$ ), 12 siblings ( $M$ age: 15.3 years; $S D$ : 2.01; range: 12.1-18.95) and 20 controls ( $M$ age: 15.27 years; $S D$ : 1.62; range: 12.29-17.62). All subjects were group-matched for age ranging between 12 and 18 years. Groups did not differ in terms of mean age $(F(2$, 64) $=1.752 ; p=0.182$ ).

The WASI (Wechsler 1999) was used to assess intellectual function. Participants with autism had mean IQ of 108.4 (SD: 16.15 ; range: $81-146$ ), siblings had mean IQ of 114.67 (SD: 10.78; range: 97-129) and controls had mean IQ of 114.05 (SD: 11.39; range: 83-131). Groups did not differ in terms of mean IQ $(F(2,64)=1.474 ; p=0.237)$. 
The project received ethical approval from the Cambridgeshire 1 Research Ethics Committee.

\section{Measures of Asymmetry}

\section{Handedness}

Handedness was assessed using the Edinburgh Handedness Inventory (EHI; Oldfield 1971), a self-completed questionnaire for determining hand preference. It consists of 10 items measuring handedness for everyday tasks such as writing, drawing, throwing, using scissors, using a toothbrush, using a knife, using a spoon, using a broom, striking a match and opening a box. Participants are asked to indicate which activity they perform exclusively with the right hand, exclusively with the left hand or with either hands. Scores are expressed as a laterality quotient using the formula "total number of items performed with the right hand - total number of items performed with the left hand/total number of items performed by both hands". Scores were treated as quantitative variables ranging from -100 indicating strongest left-handedness to +100 indicating strongest right-handedness. In total, we received 33 questionnaires from the ASC group, 10 from the sibling group and 19 from the control group (see Table 1).

\section{Image Acquisition}

MRI scans were acquired using a Siemens 3T Tim Trio scanner (Siemens Healthcare, Erlangen, Germany) at the Medical Research Council Cognition and Brain Sciences Unit, Cambridge, UK, using a high-resolution T1-weighted 3D MPRAGE (magnetization prepared rapid gradient echo) sequence with the following parameters: voxel size $1 \times 1 \times 1 \mathrm{~mm}$, repetition time $=2,250 \mathrm{~ms}$, echo time $=$ $2.99 \mathrm{~ms}$; inversion time $=900 \mathrm{~ms}$; flip angle $=9^{\circ}$; total scan time $=4 \min 16 \mathrm{~s}$ ).

\section{Image Analysis}

Image alignment and tracing measurements were performed using Analyze 10.0 (AnalyzeDirect, Inc., KS). Raters were blind to study group and all other details regarding the scans. All of the images that made up the dataset were traced by the two independent raters (DF and LRC). Before determining the corpus callosum size, all scans were spatially realigned in all three axes along the AC-PC (anterior to posterior commissure). The two blinded raters independently defined the corpus callosum for each of the 120 scans using a graphical tablet by tracing the corpus callosum in 13 consecutive sagittal slices, comprising the mid-sagittal slice and six adjacent para-sagittal slices on each side (Hardan et al. 2009).
Subsequently the corpus callosum tracings were divided into seven subregions by the two independent raters (Witelson 1989). The volume of the corpus callosum was defined as the sum of all 13 sagittal sections traced, and the volume for each subsection as the mean of the volumes from the two independent raters. Inter-rater reliabilities were calculated using the intra-class correlation coefficient (ICC).

Corpus callosum asymmetry indices (AI), comparing the total volume of the six left para-sagittal slices (LS) with the total volume of the six right parasaggital slices (RS), were calculated as: asymmetry index $(\mathrm{AI})=(\mathrm{RS}-\mathrm{LS}) /$ $(\mathrm{RS}+\mathrm{LS}) \times 100($ see Table 1$)$.

\section{Cognitive Functions}

Neuropsychological cognitive performance in terms of executive function was measured in terms of executive function with two subtests from the Cambridge Neuropsychological Test Automated Battery (CANTAB; Robbins et al. 1998) including the Intra-Dimensional/ Extra-Dimensional (ID/ED) Shift task and the Stockings of Cambridge (SOC). These tests assess cognitive flexibility and planning ability respectively, each of which is reported to be impaired in ASC (Ozonoff et al. 2004).

\section{Intra-Dimensional/Extra-Dimensional Shift (ID/ED) Task}

The ID/ED task specifically measures attentional setshifting and mental flexibility in terms of rule acquisition and reversal. Participants were asked to select a shape according to a rule. After 6 correct trials the rule changed and participants had to shift to a new rule. The task consisted of nine stages of which two constituted critical shifts. These occurred at stage six of the task when participants had to shift to a different stimulus within a relevant dimension (the "intra-dimensional" shift), and stage eight when participants had to shift attention to a new dimension (the "extra-dimensional" shift). In light of the graded nature of the task, performance is indicated by the number of stages completed, the number of trials per stage, and the number of errors made. Errors at the extra-dimensional shift stage are comparable with the category shift in the Wisconsin Card Sorting Test (Fray et al. 1996), and are considered particularly to reflect cognitive flexibility. The number of errors made at stages eight and nine was identified as the variable of interest for this analysis. Also, research indicates that especially these two stages involve prefrontal function (Dias et al. 1996).

\section{The Stockings of Cambridge (SOC) Task}

In this computerized adaptation of the Tower of London task (Shallice 1982), participants were asked to move balls 


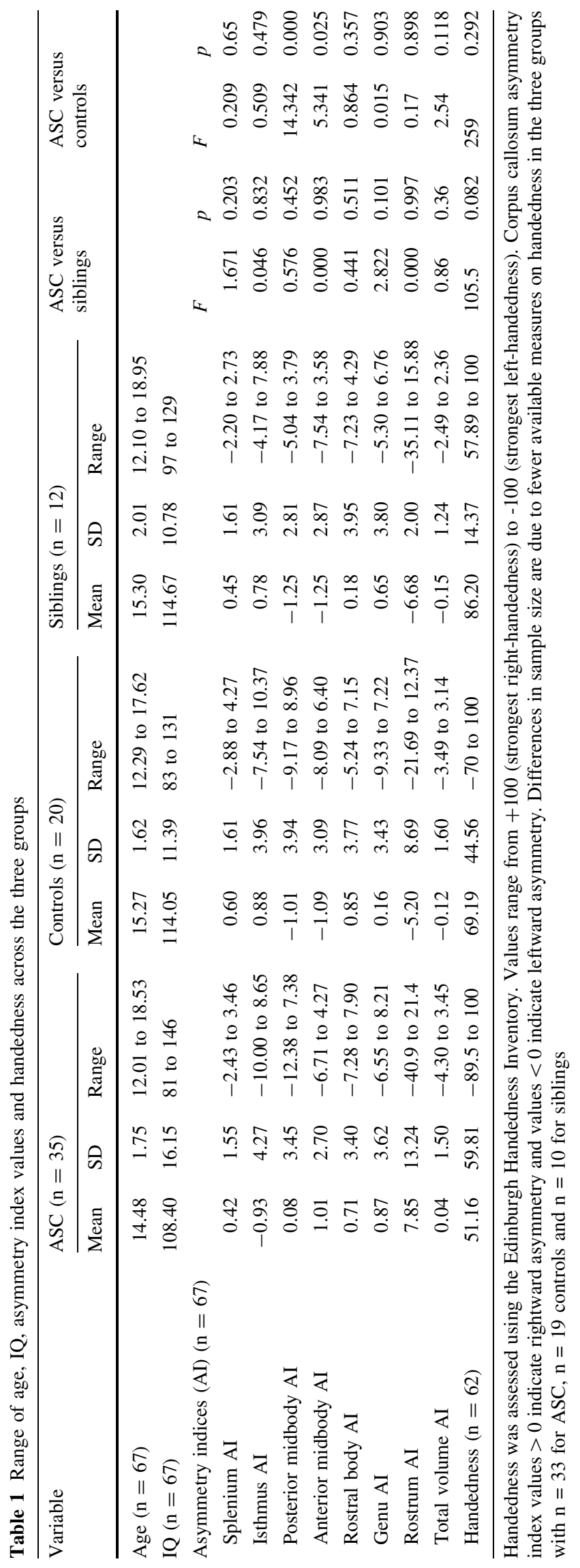


in the lower display to match a pattern in the upper display. This task increased in difficulty from 1 to 5 move problems. The mean number of moves required for completing the task measured participants' planning and problem solving ability.

\section{Symptom Severity}

Subdomains of the ADI-R (Lord et al. 1994) and the ADOS-G (Module 4) (Lord et al. 2000) were used to determine clinical symptoms in terms of abnormal social behaviour (ADI subdomain-A; ADOS subdomain-B), communication (ADI subdomain-B; ADOS subdomain-A) and stereotyped, restricted behaviour (ADI subdomain-C; ADOS subdomain-D).

Composite scores from ADOS-G and ADI-R were calculated as follows:

- Four items composed the ADOS communication score, with a range of $0-8$. Seven items composed the ADOS reciprocal social interaction score, with a range of 0-14. Four items composed the ADOS stereotyped behaviours and restricted interests score, with a range of $0-8$.

- 15 items composed the ADI qualitative abnormalities in reciprocal social interactions, with a range of 0-30. 13 items composed the ADI qualitative abnormalities in communication score, with a range of $0-26$. Six items composed the ADI restricted, repetitive and stereotyped patterns of behaviour score, with a range of $0-12$. For these scores the caregiver's descriptions of the adolescents' childhood aged 4-5 or "ever" behaviours were used.

\section{Statistical Analysis}

To assess the degree of lateralization in terms of handedness, we compared the raw Edinburgh Handedness Inventory scores of the three groups using a Kruskal-Wallis nonparametric test, as scores were not normally distributed.

We investigated corpus callosum asymmetry by a univariate analysis of covariance (ANCOVA) for AI based on total volumes, and a multivariate analysis of covariance (MANCOVA) for all AI based on the seven subregions. For both models, main effects of group and handedness were investigated along with the group-by-handedness interaction. Age was included as a covariate in both models.

Subsequently each sub-region was tested separately with an equivalent ANOCOVA to observe the profile of putative differences along the corpus callosum. Due to the greater number of comparisons involved in investigating all subregions, we sought to control for multiple comparisons by setting the $p$ value for significant at $p=0.01$. As the seven subregions are related to one another (with correlations up to $r=0.53$ ), we considered that full Bonferroni correction as $p=0.05 / 7=0.007$ may be too stringent in this case.

To identify possible endophenotypic patterns of between-group differences, we subsequently conducted between two-group tests (ASC vs. controls and siblings vs. controls) for any subregions for which significant groupby-handedness interactions were found in the above step. For these follow-up calculations between the two pairs of groups, a Bonferroni correction was applied and a level of statistical significance was set at $p=0.05 / 2=0.025$.

The relationship between corpus callosum asymmetry and cognitive function was investigated in separate ANCOVAs with full scale IQ and the mean number of moves in the SOC task as the dependent variables and in a MANCOVA with total number of errors made in stages eight and nine on the ID/ED task as the dependent variables. In all three models, main effects of group and AI along with the group-by-AI interaction were investigated covarying for age. For these calculations, the $p$ value was not corrected, as the two stages on the ID/ED task are highly inter-correlated $(r=0.81, p<0.001)$ and thus the tests are not independent of each other.

Similar to the analyses above, group-by-AI interaction was subsequently tested for an endophenotype using two group tests: ASC versus controls and siblings versus controls. Bonferroni correction was applied; $p=0.05 / 2=$ 0.025 .

To explore any relationship between $\mathrm{AI}$ and symptoms, within-group partial correlation (controlling for age) was undertaken between corpus callosum asymmetry measures and clinical symptom severity as measured by composite measures of the ADOS-G and ADI-R for the ASC group only.

In the above analyses, significant main effects or interactions found were further investigated using correlation analysis.

\section{Results}

Handedness

There was no significant overall effect of group across the three groups $(H(2)=3.313 ; p=0.191)$. Also, participants with ASC did not differ significantly from their unaffected siblings $(U=105.5 ; z=-1.741 ; p=0.082 ; r=-0.27)$ or from controls $(\mathrm{U}=259 ; \mathrm{z}=-1.054 ; p=0.292 ; r=$ -0.15 ) (see Fig. 2). We then applied a quartile split in order to test whether the groups were differently distributed across the handedness spectrum, without any significant effects 


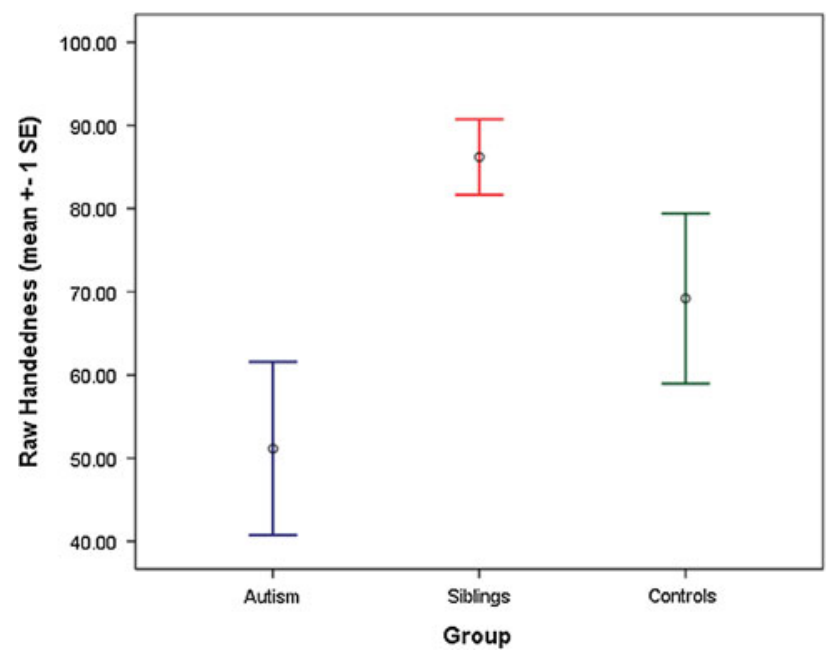

Fig. 2 Distribution of raw handedness scores as measured by the Edinburgh Handedness Inventory ranging from +100 (strong righthandedness) to -100 (strong left-handedness) in the 3 groups

$(25 \%: H(1)=0.097 ; p=0.755 ; 50 \%: H(2)=1.028$; $p=0.598 ; 75 \% / 100 \%: H(2)=0.95 ; p=0.622)$.

As the sample size was less than 25 in the sibling and control group, we calculated in addition the KolmogorovSmirnov Z. We found a trend towards a significant difference between the ASC and sibling group $(Z=1.318$; $p=0.062$ ), with the ASC group being less consistently right-handed, but no significant difference between the ASC and control group $(Z=0.72 ; p=0.678)$.

\section{Asymmetry of the Corpus Callosum}

\section{Relationship to Lateralization (Handedness)}

Inter-rater reliability was calculated for all corpus callosum volume measurements: ICC $=0.98$ for the total volume; ICC $=0.99$ for the splenium; ICC $=0.97$ for the isthmus; ICC $=0.96$ for the posterior midbody; ICC $=0.97$ for the anterior midbody; ICC $=0.99$ for the rostral body; ICC $=0.97$ for the genu; ICC $=0.97$ for the rostrum.

In terms of asymmetry, we performed an ANCOVA for AI derived from global volume and a MANCOVA for AI based on the seven subregions with age as a covariate exploring main effects and group-by-handedness interaction. For AI from global volume groups did not differ significantly from each other based on handedness $(F(2$, $55)=1.806 ; p=0.174)$. For AIs from the subregions of the corpus callosum, the group-by-handedness MANCOVA interaction bordered on significance (Wilk's lambda $=0.637 ; F(14,98)=1.774 ; p=0.053)$.

Separate univariate ANCOVAS for each sub-region showed a significant interaction between group and

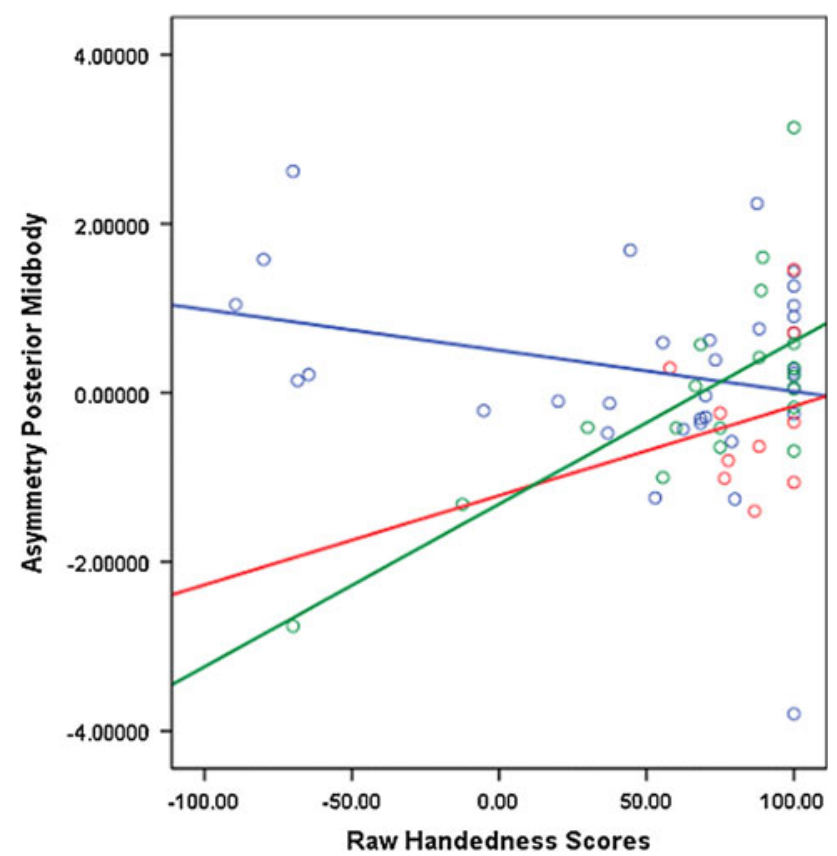

Fig. 3 Asymmetry of the posterior midbody in standardized residual units (with age regressed out) in relation to handedness across the three groups. Values $>0$ indicate rightward asymmetry and values $<0$ indicate leftward asymmetry. Blue line ASC, red line siblings and green line controls (Color figure online)

handedness for the posterior midbody $(F(2,55)=7.572$; $p=0.001)$. The anterior midbody $(F(2,55)=2.601$; $p=0.083)$ and genu $(F(2,55)=2.614 ; p=0.082)$ showed a marginal trend towards significance.

Two-group analyses revealed that group-by-handedness interaction for ASC versus control was significant in the posterior midbody $(F(1,47)=14.342 ; p<0.001)$ with significant main effects of both group $(F(1,47)=11.747$; $p=0.001)$ and handedness $(F(1,47)=5.271 ; p=0.026)$ (see Fig. 3) and for the anterior midbody $(F$ (1, $47)=5.341 ; p=0.025)$ with a significant main effect of handedness $(F(1,47)=5.019 ; p=0.03)$ (see Fig. 3). There were no significant group-by-handedness interactions in the siblings versus controls comparison (posterior midbody: $F(1,24)=0.004 ; p=0.953$; anterior midbody: $F(1,24)=0.001 ; p=0.972)$.

Asymmetry index values of the posterior midbody were positively correlated with handedness in the control group $(r=0.561 ; p=0.013)$, indicating that rightward asymmetry of these subregions was associated with a rightward shift in handedness. The same association was marginally significant for the anterior midbody $(r=0.417$; $p=0.076)$. These correlations were not significant in the ASC group (posterior midbody: $r=0.017 ; p=0.926$; anterior midbody: $r=0.02 ; p=0.912$ ) and the sibling group (posterior midbody: $r=0.113 ; p=0.757$; anterior midbody: $r=-0.069 ; p=0.85$ ). 


\section{Relationship to Cognitive Function}

For IQ the ANCOVA revealed a significant main effect of rostral asymmetry with stronger rightward asymmetry being related to higher IQ $(F(1,60)=6.04 ; p=0.017)$. Subsequent partial correlations with age as a covariate, conducted separately by group, revealed that the association was only significant in the ASC group $(r=0.526$; $p=0.001)$, but not in the siblings $(r=0.205 ; p=0.546)$ or controls $(r=0.243 ; p=0.316)$.

For the ID/ED, the only significant interaction occurred between group and asymmetry index values of the isthmus for stage eight $(F(2,61)=3.338 ; p=0.042)$ across all three groups. Follow-up analyses revealed that this interaction was significant between the ASC and control group for the number of errors made in stage eight $(F$ (1, $51)=7.481 ; p=0.009)$ with a significant main effect of group $(F(1,51)=6.58 ; p=0.013)$. The interaction was not significant across siblings and controls (stage eight: $F(1,28)=2.497 ; p=0.125)$.

Subsequently conducted correlations (separate by group) between stage eight of the ID/ED task and asymmetry values of the isthmus were significant in both the ASC group $(r=-0.371 ; p=0.028)$ and the control group $(r=0.633 ; p=0.003)$, but not the sibling group $(r=$ $-0.174 ; p=0.588)$.

For the SOC task, the interaction between group and corpus callosum asymmetry did not reveal any significant results.

\section{Relationship to Clinical Symptom Severity}

Bivariate partial correlations (accounting for age) between AI and symptom severity were carried out only in the ASC group. We found that asymmetry of the splenium was positively correlated with symptom severity scores for social interaction difficulties (subdomain-B) $(r=0.358$; $p=0.038$ ) on the ADOS-G. Also, asymmetry of the posterior midbody was significantly positively correlated with symptom severity scores for repetitive and stereotyped behaviours (subdomain-D) $(r=0.411 ; p=0.016)$ on the ADOS-G. In both cases, greater rightward asymmetry was associated with a greater severity of symptom scores. Also, rightward asymmetry of the rostral body was associated with higher scores for abnormalities in reciprocal social interaction (subdomain-A) on the ADI-R $(r=0.390$; $p=0.023$ ). Asymmetry of the rostrum was significantly negatively correlated with symptom severity scores for social communication (subdomain B) as measured by the ADI-R $(r=-0.406 ; p=0.017)$, with greater leftward asymmetry being associated with greater severity of symptoms.

\section{Discussion}

This study tested whether ASC was related to an atypical pattern of hemispheric lateralization, both in terms of functional and neuroanatomical asymmetry.

\section{Corpus Callosum Asymmetry}

We found significant interactions between group and handedness for the posterior and the anterior midbody between participants with ASC and controls. Both results implied that left-handed controls were more strongly leftward lateralized than left-handed adolescents with ASC in both subregions. In the control group, but not the ASC group, this correlation between handedness and asymmetry was significant.

Luders et al. (2006) found similar results showing stronger rightward lateralization in the anterior callosal section which projects to the motor cortices in right-handed males, concluding that stronger leftward lateralization of motor functions in right-handers reduces left interhemispheric fibres. Our findings are consistent with this and reports suggesting that motor transfer is more efficient from the right to the left hemisphere via the corpus callosum (Braun et al. 2003; Saron et al. 2003). We did not find this relationship between handedness and asymmetry in the ASC group. As the lack of an association does not necessarily imply impairment, we can just speculate that the absence of the normally occurring leftward lateralization in left-handed people with ASC might result in a less favourable distribution of commissural connections.

The corpus callosum has been found to play an important role in the neuropathology of autism. However, most studies have only looked at its size and there are no existing results on asymmetry. We therefore can only compare our findings to other groups of people with brain lesions in these callosal regions or indirectly via looking at injuries in cortical areas, which the callosal subregions carry projections to. The anterior midbody projects to the motor cortex (Hofer and Frahm 2006), while the posterior midbody projects to the posterior parietal cortex (PPC), which is a highly interconnected region integrating motor and sensory input from somatic and visual modalities (Andersen and Buneo 2002). It subserves higher cognitive functions such as early planning of sensory-guided movements (such as eye, grasp, and reach movements) and regulation of controlled behaviour in response to environmental stimuli. Studies looking at behavioural and cognitive outcomes in patients with lesions in the middle and posterior part of the corpus callosum report deficits in complex bimanual coordinative movements (Meyer et al. 1998) and interhemispheric transfer of tactile information (Bentin et al. 1984). Patients with PPC lesions display difficulties in 
following verbal commands, performing sequences of actions and fine-tuning eye and hand movements when grasping things (Geschwind and Damasio 1985; Goodale and Milner 1992; Perenin and Vighetto 1988). Similar problems in ASC suggests that atypical lateralization of the posterior midbody and the anterior midbody might underpin symptoms such as clumsiness (Burgoine and Wing 1983; Ghaziuddin and Butler 1998), impaired fine motor skills (Dawson and Watling 2000), difficulties in motor planning and planned sequencing of actions (Greenspan and Wieder 1997) and difficulties responding to spoken commands (Preis 2006). As one sub-region of the PPC is involved in the planning of eye-movements, the question arises whether anomalous joint attention and eye contact in autism may be related to this brain region as well.

In a case study, Dimond et al. (1977) report behavioural, verbal and emotional deficits following section of the middle third of the corpus callosum. After surgery impairments were present in linguistic ability characterized by simplified or repetitive and stereotyped language, alongside difficulties in expressing and naming emotions, and a change in ear preference on the dichotic listening task as well as in hand-preference in favour of the left. The similarities in symptomatology to autism are striking. Also, reports of complete agenesis of the corpus callosum confirm that associated symptoms such as impaired social skills overlap with the diagnostic criteria for autism (Badaruddin et al. 2007; Paul et al. 2007). We have to be careful when deriving conclusions from these studies for our own, as we don't know if atypical asymmetries and brain lesions are comparable on a neuroanatomical level in terms of clinical outcome. Still, morphological alterations in the same region might lead to similar cognitive deficits.

Previous studies have looked at the relationship between handedness and the corpus callosum showing that nonconsistently right-handed men have a larger corpus callosum than consistently right-handed men (Witelson 1989). This consistently reported finding seems incompatible with the notion that individuals with ASC are more often reported to be left-handed and to have reduced corpus callosum volume. Taking both direction (right vs. left) and degree (consistent vs. non-consistent) of lateralization into account, the non-consistently right-handed individuals might actually represent a group of people with greater bihemispheric cognitive representation. This would be in line with findings showing that less strongly lateralized people, such as women, have a larger corpus callosum (Steinmetz et al. 1995). In the same way, Luders et al. (2010) found that less lateralized left-handers had a larger corpus callosum than strongly lateralized right-handers and so did less lateralized right-handers in comparison to strongly lateralized left-handers.
Future studies should further explore how the direction and the degree of handedness differentially relate to callosal size and asymmetry in autism in comparison to typical individuals. It has yet to be defined whether lefthanded individuals with autism compose a qualitatively different group, with possibly different degrees of lateralization. Looking at callosal asymmetries in this context can give us even more detailed information on the location of callosal size reductions or increases and shed also light on the degree of lateralization in related cortical areas.

\section{Handedness}

We did not find an overall difference in handedness between the three groups. Participants with ASC did not differ from controls in handedness, in contrast to previous studies which have documented greater functional rightlateralization. However, some studies have failed to establish significant differences in handedness between people with ASC and controls (Barry and James 1978) or also found significant differences between people with ASC and their siblings or parents (Boucher 1977; Tsai 1982). Incongruity in results might partly be due to methodological limitations. There are many discrepancies in handedness assessment across different studies, with some only asking subjects to specify their writing hand (Vuoksimaa et al. 2010), while others ask them to fill in different questionnaires (Annett 1970; Crovitz and Zener 1962; Oldfield 1971) or perform manual tasks (Hand Preference Demonstration Task; Soper et al. 1986). Also, studies differ in the way handedness is classified, and where cut-off points for categories are set, resulting in classifications such as "left- versus right-handedness", "right- versus non-right-handedness", and "mixed handedness". Many report neither IQ nor age, which is important to specify the level of cognitive functioning and to assess the possibility of whether mixed-handedness reflects an age-related developmental lag.

Due to the small number of available handedness measures in the sibling $(n=10)$ and control group $(n=19)$, the power to detect handedness differences might have been limited. In fact, the effect sizes for the handedness difference between the ASC and sibling group $(r=-.27)$ and the ASC and control group $(r=-0.15)$ both are very small. Additionally, non-parametric tests are known to be less powerful than parametric tests. The trend in the data in the expected direction suggests that with additional numbers the between group differences may reach significance, consistent with previous reports.

In view of the large number of consistent reports, it seems that there is a shift in the handedness distribution towards non-right-handedness in ASC. The question remaining is what these elevated rates tells us. In contrast 
to neuroanatomical asymmetries, handedness appears to be a more global marker of lateralization and due to the lack of a neurobiological substrate, it is less straightforward to interpret. In order to be able to derive conclusions, it is important to look at it in association with other traits with known brain structural correlates such as language or cognitive function, which can be impaired in autism. Accordingly, as we did not find a marked difference between our overall sample of adolescents with ASC and controls, the question arises, whether there are differences in lateralization between certain subgroups within people with ASC based on their level of functioning. DeLong (1978), for example, argues that there are different handedness subtypes in autism comprising a "bihemispheric autistic syndrome" which is related to lower levels of functioning and mixed handedness and a "left hemisphere autistic syndrome" which is less severe and linked to high-functioning autism. In line with this, several studies report that lower functioning individuals may be more likely to display mixed-handedness (Fein et al. 1984; Tsai 1983).

Our ASC group seemed to be less strongly lateralized compared to the two other groups. The EHI score is not sensitive to differentiate between ambiguous and ambidextrous handedness patterns, so that this less strongly lateralized pattern in the autism group could mean that they use different hands for different tasks (ambidextrous) or different hands for same tasks (ambiguous). The latter would reflect that they are unable to establish a normal pattern of lateralization, which makes them switch hands within tasks. However, as ambiguous handedness has been linked to low functioning autism and as we only included individuals with IQ scores higher than 70, it is less likely that this applies to our participants. Future research would benefit from using tests that distinguish between these two patterns and from comparing low-functioning with highfunctioning individuals to establish the relationship between handedness and the level of cognitive functioning.

Also, Escalante-Mead et al. (2003) consider diagnostic sub-groups separately. They reported reduced rates of established lateral preference in individuals with autism with delayed language development compared to individuals with autism with typical early language acquisition. We did not distinguish between different subgroups, as we did not look at Asperger Syndrome and HFA separately in our analyses.

These considerations are consistent with the idea that atypical handedness is a marker of different etiologic subgroups within ASC. Future studies should differentiate between subtypes within ASC, and include measures such as age and IQ, alongside consistent handedness classifications, to establish whether different patterns of lateralization manifest at different degrees of impairment.

\section{Cognitive Function and Symptom Severity}

Strikingly, we found a significant correlation between leftward asymmetry of the isthmus and worse performance on stage eight of the ID/ED task in individuals with autism. Based on Bradshaw's fronto-striatal model (2001), we would have expected that rightward asymmetry of regions projecting to the frontal lobe, such as the rostral body, genu or rostrum would show an association with poorer executive function. An association with one of these regions has been found by Just et al. (2007) showing that size reductions in the genu were associated with functional underconnectivity between frontal and parietal areas during the Tower of London task. Still, also Keary et al. (2009) report that worse performance in participants with autism on the Tower of Hanoi and Wisconsin card sorting test was associated with smaller isthmus size. As this region projects to the tempo-parietal cortex, which is involved in language and complex cognitive functions, its role in executive function has yet to be established. Since this was the only result for the ID/ED task and there were no results for the SOC task, we assume that atypical lateralization might play a less crucial role for executive function deficits. Nonetheless, further research is needed to confirm this.

Rightward asymmetry of several callosal subregions such as the splenium, the rostral body and the posterior midbody were associated with increased symptom severity. The splenium has projections to the occipital and inferior temporal lobe that are crucial for face and object processing. Schultz (2005) has shown unusually greater activity in the inferior temporal gyrus and less activity in the fusiform face area (FFA) during face processing in autism compared to controls. Difficulties with face processing in autism constitute an underlying precursor of social interaction difficulties and might also provide a potential explanation for why alterations in the splenium region are associated with social interaction difficulties as measured by the ADOS subdomain-B.

Rightward asymmetry of the rostral body was related to more marked abnormalities in reciprocal social interactions as measured by the ADI subdomain-A. This is in line with Bradshaw's fronto-striatal model (2001) stating that abnormally lateralized frontal circuits constitute the base for impairments in higher cognitive and social functions including social behaviour.

Rightward asymmetry of the posterior midbody was associated with stronger symptom severity as measured by the ADOS subdomain-D. As described previously, the PPC integrates sensory and motor input. Autism is sometimes associated with sensory processing abnormalities in terms of hypo- or hyper-responsiveness to sensory input and sensory processing abnormalities have been found to be correlated with stereotyped and repetitive behaviour 
(Baranek et al. 1997). We found rightward asymmetry of the posterior midbody to be associated with stronger repetitive, stereotyped behaviour, which might suggest an indirect corroboration of that association. This implies that rightward lateralization (and possibly under-connectivity) may disturb the normal process of planned movement generation and result in stereotyped patterns of behaviour and body mannerisms.

Contrary to the other results, asymmetry of the rostrum did not show rightward disadvantage. Rostral rightward asymmetry was instead associated with higher IQ scores and less severe communication impairment. This is particularly striking as this region is thought to connect to prefrontal areas and it has been suggested that dysfunction of the left fronto-striatal system relates to deficits in higherorder cognitive functions in autism (Bradshaw 2001). This raises the question of whether not only impairments in high functioning autism, but also strengths of the condition such as high IQ or good systemizing skills, might be related to the same atypical pattern of lateralization in the same way. Since these two domains (IQ and onset of language) set Asperger Syndrome apart from classic autism, it would be interesting to test in future research whether there is a difference in cerebral lateralization between the two diagnostic sub-groups. Even though it is plausible to suggest that as the rostrum is the smallest sub-region of the corpus callosum it may be difficult to define, remarkably high inter-rater-reliability should substantiate the accuracy of our measurement.

\section{Broader Autism Phenotype}

In terms of callosal asymmetry siblings showed an intermediate position between the two other groups for the posterior and anterior midbody in relation to hand-preference. The same pattern occurred for neuropsychological performance based on isthmus asymmetry. However, the difference between siblings and controls was not significant for either result. Also in terms of handedness, participants with ASC showed a more pronounced difference to their siblings than to controls. These findings lead us to assume that cerebral lateralisation does not appear to be a marker of familial risk for the condition implicating that either environmental factors have considerable influence on the establishment of lateralization or that individuals with autism carry genetic variants with increased vulnerability for developing the condition that are not shared by their siblings.

In accordance with these findings, a recently published study on the genetic liability for autism showed that the relative influence of shared environment in twin pairs was higher than genetic heritability (Hallmayer et al. 2011). They raise the issue that genetic factors have been overestimated in previous reports and environmental influence should be taken into account to a greater extent in future research.

Some studies corroborate that genetic influence towards left- handedness is rather limited. Tsai (1982) found that children with autism significantly differed from their siblings and parents displaying stronger non-right-handedness. Also Boucher et al. (1990) reported similar findings showing that parents of children with autism had an increased incidence of pure right-handedness compared to their children. In terms of heritability of callosal morphology, Scamvougeras et al. (2003) found corpus callosum volume to be highly heritable in young typical men and women, with a genetic influence of $94 \%$. Nevertheless, other studies report that the corpus callosum is one of the last main fibre tracts to mature in humans and its functional maturation extends into late childhood (Rakic and Yakovlev 1968). Thus, reports on environmentally induced callosal size differences (Lee et al. 2003; Sanchez et al. 1998; Schlaug et al. 2009, 1995) suggest that certain environmental stimuli could possibly affect corpus callosum morphology and patterns of cerebral lateralization.

Underlying mechanisms causing cerebral lateralization are still poorly understood, but as genetic studies of the developmental origins of handedness have shown, only $25 \%$ of its variability is explained by genetic influences (Medland et al. 2006). It is believed to be influenced to some extent by factors such as sex (Gilbert and Wysocki 1992), age (Ellis et al. 1998), disruptive events in pregnancy (Bakan et al. 1973) and prenatal androgen levels (Tan 1991). In particular the latter has been suggested as a potential factor in contributing to the establishment of functional brain lateralization, as handedness originates prenatally (Hepper et al. 1998; Witelson 1987), and males are more often left-handed than females (Annett 1970; Papadatou-Pastou et al. 2008). Accordingly, exposure to high levels of testosterone during critical periods of foetal brain development might lead to an unequal brain development enhancing the maturation of the right hemisphere and resulting in non-right-handedness (Geschwind and Galaburda 1985a, b, c). We have previously shown that prenatal testosterone is associated with rightward asymmetry of one region of the corpus callosum (Chura et al. 2010) and with asymmetry of specific grey matter regions (Lombardo et al. 2012). Future research is required to explore whether prenatal testosterone is associated with cerebral lateralization.

\section{Conclusions}

It appears that callosal asymmetries are not necessarily negative in general. As seen in our controls and in the study 
of Luders et al. (2006), rightward asymmetry of anterior regions seems to be a normal pattern in right-handed men. It would not be surprising if other studies also found asymmetries in callosal subsections projecting to language regions such as the splenium or isthmus. In the light of these considerations, there are several explanations that could account for cerebral asymmetries seen in autism. One is the commonly cited left hemisphere dysfunction theory stating that atypical global rightward asymmetries result in autism-related deficits. Since there is also normally occurring rightward lateralization in the cerebral cortex in typical people, another possible explanation is that individuals with ASC exhibit reversed patterns of normal asymmetry, which can occur in either direction. A last possibility is that people with ASC show the same directional asymmetries as typical people, but might differ in quantitative terms with an atypically greater amount of asymmetry.

In our study, adolescents with ASC did not differ from controls in terms of functional asymmetry (handedness), but neuroanatomically they showed reversed patterns of the associations between the posterior midbody and anterior midbody and handedness on the one hand, and between the isthmus and executive function on the other hand. Most neuroanatomical asymmetry measures indicated that rightward lateralization was associated with stronger symptom severity. We could not compare these findings to the sibling or control group since they were based on symptoms only present in autism. Thus, for this result, we cannot establish whether the underlying neuroanatomical alteration is of qualitative or quantitative nature.

As seen in the isthmus and rostrum, we could not fully support the left hemisphere dysfunction theory, as we did not find a consistent picture of that cognitive disadvantage was restricted to rightward lateralization. An important question for future research consequently is whether the left hemisphere dysfunction theory of autism is too broad an approach and whether depending on the region being investigated, different patterns of lateralization might be beneficial.

As siblings did not show a similar pattern to the autism group in handedness and were not significantly different from controls in terms of callosal asymmetry (based on handedness) and executive function (based on callosal asymmetry), we suggest that atypical asymmetry may be a marker of the condition itself rather than of a familial risk in the broader phenotype.

In this study we only had sufficient numbers of male participants and therefore could not assess whether similar findings are present in females with ASC.

We only measured the asymmetry of the corpus callosum, however its topographic organization indirectly implicates other cortical areas as potentially contributing to the neuroanatomical basis of autism. As it also includes heterotopic callosal connections to non-corresponding cortical areas, future work should relate asymmetry measures of other brain regions to cognitive functions and symptom severity in autism.

Acknowledgments We are grateful to all participants and their families for their participation in our study and to all autism supportorganizations that helped with recruitment. This research was funded by an MRC Clinician Scientist Fellowshipto MDS from the UK Medical Research Council (G0701919). This study was conducted in association withNIHR CLAHRC for Cambridgeshire and Peterborough NHS Foundation Trust. We are grateful to Dr. MengChuan Lai for comments and helpful advice.

\section{References}

Aboitiz, F., Scheibel, A. B., Fisher, R. S., \& Zaidel, E. (1992). Individual differences in brain asymmetries and fiber composition in the human corpus callosum. Brain Research,598(1-2), 154-161.

Allen, L. S., Richey, M. F., Chai, Y. M., \& Gorski, R. A. (1991). Sex differences in the corpus callosum of the living human being. Journal of Neuroscience,11(4), 933-942.

Andersen, R. A., \& Buneo, C. A. (2002). Intentional maps in posterior parietal cortex. Annual Review of Neuroscience, 25, 189-220.

Annett, M. (1970). A classification of hand preference by association analysis. British Journal of Psychology,61(3), 303-321.

Badaruddin, D. H., Andrews, G. L., Bolte, S., Schilmoeller, K. J., Schilmoeller, G., Paul, L. K., et al. (2007). Social and behavioral problems of children with agenesis of the corpus callosum. Child Psychiatry and Human Development,38(4), 287-302.

Bailey, A., Le Couteur, A., Gottesman, I., Bolton, P., Simonoff, E., Yuzda, E., et al. (1995). Autism as a strongly genetic disorder: Evidence from a British twin study. Psychological Medicine, 25(1), 63-77.

Bakan, P., Dibb, G., \& Reed, P. (1973). Handedness and birth stress. Neuropsychologia,11(3), 363-366.

Baranek, G. T., Foster, L. G., \& Berkson, G. (1997). Tactile defensiveness and stereotyped behaviors. American Journal of Occupational Therapy,51(2), 91-95.

Barry, R. J., \& James, A. L. (1978). Handedness in autistics, retardates, and normals of a wide age range. Journal of autism and childhood schizophrenia,8(3), 315-323.

Belmonte, M. K., Allen, G., Beckel-Mitchener, A., Boulanger, L. M., Carper, R. A., \& Webb, S. J. (2004). Autism and abnormal development of brain connectivity. Journal of Neuroscience,24(42), 9228-9231.

Bentin, S., Sahar, A., \& Moscovitch, M. (1984). Intermanual information transfer in patients with lesions in the trunk of the corpus callosum. Neuropsychologia,22(5), 601-611.

Berg, E. A. (1948). A simple objective technique for measuring flexibility in thinking. Journal of General Psychology,39, 15-22.

Berument, S. K., Rutter, M., Lord, C., Pickles, A., \& Bailey, A. (1999). Autism screening questionnaire: Diagnostic validity. British Journal of Psychiatry, 175, 444-451.

Blackstock, E. G. (1978). Cerebral asymmetry and the development of early infantile autism. Journal of autism and childhood schizophrenia,8(3), 339-353.

Borys, S. V., Spitz, H. H., \& Dorans, B. A. (1982). Tower of Hanoi performance of retarded young adults and nonretarded children as a function of solution length and goal state. Journal of Experimental Child Psychology,33(1), 87-110. 
Boucher, J. (1977). Hand preference in autistic children and their parents. Journal of autism and childhood schizophrenia,7(2), 177-187.

Boucher, J., Lewis, V., \& Collis, G. (1990). Hand dominance of parents and other relatives of autistic children. Developmental Medicine and Child Neurology,32(4), 304-313.

Bradshaw, J. L. (2001). Developmental disorders of the frontostriatal system: Neuropsychological, neuropsychiatric and evolutionary perspectives. Hove: Psychology Press/Taylor and Francis.

Brambilla, P., Hardan, A., di Nemi, S. U., Perez, J., Soares, J. C., \& Barale, F. (2003). Brain anatomy and development in autism: Review of structural MRI studies. Brain Research Bulletin,61(6), 557-569.

Braun, C. M. J., Achim, A., \& Larocque, C. (2003). The evolution of the concept of interhemispheric relay time (the parallel brain). Cambridge, MA: MIT Press.

Bryson, S. E. (1990). Autism and anomalous handedness (left handedness). Amsterdam: Elsevier.

Burgoine, E., \& Wing, L. (1983). Identical triplets with Asperger's syndrome. British Journal of Psychiatry, 143, 261-265.

Chiron, C., Leboyer, M., Leon, F., Jambaque, I., Nuttin, C., \& Syrota, A. (1995). SPECT of the brain in childhood autism: Evidence for a lack of normal hemispheric asymmetry. Developmental Medicine and Child Neurology,37(10), 849-860.

Chura, L. R., Lombardo, M. V., Ashwin, E., Auyeung, B., Chakrabarti, B., Bullmore, E. T., et al. (2010). Organizational effects of fetal testosterone on human corpus callosum size and asymmetry. Psychoneuroendocrinology,35(1), 122-132.

Clarke, J. M., \& Zaidel, E. (1994). Anatomical-behavioral relationships: Corpus callosum morphometry and hemispheric specialization. Behavioural Brain Research,64(1-2), 185-202.

Colby, K. M., \& Parkison, C. (1977). Handedness in autistic children. Journal of autism and childhood schizophrenia,7(1), 3-9.

Cornish, K. M., \& McManus, I. C. (1996). Hand preference and hand skill in children with autism. Journal of Autism and Developmental Disorders,26(6), 597-609.

Courchesne, E., \& Pierce, K. (2005). Why the frontal cortex in autism might be talking only to itself: Local over-connectivity but longdistance disconnection. Current Opinion in Neurobiology, 15(2), $225-230$.

Crovitz, H. F., \& Zener, K. (1962). A group-test for assessing handand eye-dominance. American Journal of Psychology,75, 271-276.

Dawson, G., \& Watling, R. (2000). Interventions to facilitate auditory, visual, and motor integration in autism: A review of the evidence. Journal of Autism and Developmental Disorders,30(5), 415-421.

De Fosse, L., Hodge, S. M., Makris, N., Kennedy, D. N., Caviness, V. S., Jr, McGrath, L., et al. (2004). Language-association cortex asymmetry in autism and specific language impairment. Annals of Neurology,56(6), 757-766.

DeLong, G. R. (1978). A neuro-psychological interpretation of infantile autism (Autism: A reappraisal of concepts and treatment). New York: Plenum Press.

Dias, R., Robbins, T. W., \& Roberts, A. C. (1996). Dissociation in prefrontal cortex of affective and attentional shifts. Nature,380(6569), 69-72.

Dimond, S. J., Scammell, R. E., Brouwers, E. Y., \& Weeks, R. (1977). Functions of the centre section (trunk) of the corpus callosum in man. Brain,100(3), 543-562.

Dorion, A. A., Chantome, M., Hasboun, D., Zouaoui, A., Marsault, C., Capron, C., et al. (2000). Hemispheric asymmetry and corpus callosum morphometry: A magnetic resonance imaging study. Neuroscience Research,36(1), 9-13.

Ellis, S. J., Ellis, P. J., Marshall, E., Windridge, C., \& Jones, S. (1998). Is forced dextrality an explanation for the fall in the prevalence of sinistrality with age? A study in northern England. Journal of Epidemiology and Community Health,52(1), 41-44.

Escalante-Mead, P. R., Minshew, N. J., \& Sweeney, J. A. (2003). Abnormal brain lateralization in high-functioning autism. Journal of Autism and Developmental Disorders,33(5), 539-543.

Fein, D., Humes, M., Kaplan, E., Lucci, D., \& Waterhouse, L. (1984). The question of left hemisphere dysfunction in infantile autism. Psychological Bulletin,95(2), 258-281.

Folstein, S. E., \& Rosen-Sheidley, B. (2001). Genetics of autism: Complex aetiology for a heterogeneous disorder. Nature Reviews Genetics,2(12), 943-955.

Folstein, S. E., \& Rutter, M. (1977). Infantile autism: A genetic study of 21 twin pairs. Journal of Child Psychology and Psychiatry, 18(4), 297-321.

Foong, J., Maier, M., Clark, C. A., Barker, G. J., Miller, D. H., \& Ron, M. A. (2000). Neuropathological abnormalities of the corpus callosum in schizophrenia: A diffusion tensor imaging study. Journal of Neurology, Neurosurgery and Psychiatry,68(2), 242-244.

Fray, P. J., Robbins, T. W., \& Sahakian, B. J. (1996). Neuropsychiatric applications of CANTAB. International Journal of Geriatric Psychiatry, 11, 329-336.

Galaburda, A. M., Rosen, G. D., \& Sherman, G. F. (1990). Individual variability in cortical organization: Its relationship to brain laterality and implications to function. Neuropsychologia,28(6), 529-546.

Geschwind, N., \& Damasio, A. R. I. (1985). Apraxia (Handbook of Clinical Neurology). Amsterdam: Elsevier.

Geschwind, N., \& Galaburda, A. M. (1985a). Cerebral lateralization. Biological mechanisms, associations, and pathology: I. A hypothesis and a program for research. Archives of Neurology, 42(5), 428-459.

Geschwind, N., \& Galaburda, A. M. (1985b). Cerebral lateralization. Biological mechanisms, associations, and pathology: II. A hypothesis and a program for research. Archives of Neurology, $42(6), 521-552$.

Geschwind, N., \& Galaburda, A. M. (1985c). Cerebral lateralization. Biological mechanisms, associations, and pathology: III. A hypothesis and a program for research. Archives of Neurology, 42(7), 634-654.

Ghaziuddin, M., \& Butler, E. (1998). Clumsiness in autism and Asperger syndrome: A further report. Journal of Intellectual Disability Research,42(Pt 1), 43-48.

Giedd, J. N., Castellanos, F. X., Rajapakse, J. C., Vaituzis, A. C., \& Rapoport, J. L. (1997). Sexual dimorphism of the developing human brain. Progress in Neuro-Psychopharmacology and Biological Psychiatry,21(8), 1185-1201.

Gilbert, A. N., \& Wysocki, C. J. (1992). Hand preference and age in the United States. Neuropsychologia,30(7), 601-608.

Gillberg, C. (1983). Autistic children's hand preferences: Results from an epidemiological study of infantile autism. Psychiatry Research,10(1), 21-30.

Goodale, M. A., \& Milner, A. D. (1992). Separate visual pathways for perception and action. Trends in Neurosciences, 15(1), 20-25.

Greenspan, S. I., \& Wieder, S. (1997). Developmental patterns and outcomes in infants and children with disorders in relating and communicating: A chart review of 200 cases of children with autistic spectrum diagnoses. The Journal of Developmental and Learning Disorders, 1(1), 87-114.

Hallmayer, J., Cleveland, S., Torres, A., Phillips, J., Cohen, B., Torigoe, T., et al. (2011). Genetic heritability and shared environmental factors among twin pairs with autism. Archives of General Psychiatry,68(11), 1095-1102.

Hardan, A. Y., Minshew, N. J., \& Keshavan, M. S. (2000). Corpus callosum size in autism. Neurology,55(7), 1033-1036. 
Hardan, A. Y., Pabalan, M., Gupta, N., Bansal, R., Melhem, N. M., Fedorov, S., et al. (2009). Corpus callosum volume in children with autism. Psychiatry Research,174(1), 57-61.

Hashimoto, T., Sasaki, M., Fukumizu, M., Hanaoka, S., Sugai, K., \& Matsuda, H. (2000). Single-photon emission computed tomography of the brain in autism: Effect of the developmental level. Pediatric Neurology,23(5), 416-420.

Hauser, S. L., DeLong, G. R., \& Rosman, N. P. (1975). Pneumographic findings in the infantile autism syndrome. A correlation with temporal lobe disease. Brain,98(4), 667-688.

Hepper, P. G., McCartney, G. R., \& Shannon, E. A. (1998). Lateralised behaviour in first trimester human foetuses. Neuropsychologia,36(6), 531-534.

Herbert, M. R., Harris, G. J., Adrien, K. T., Ziegler, D. A., Makris, N., Kennedy, D. N., et al. (2002). Abnormal asymmetry in language association cortex in autism. Annals of Neurology,52(5), $588-596$.

Hier, D. B., LeMay, M., \& Rosenberger, P. B. (1979). Autism and unfavorable left-right asymmetries of the brain. Journal of Autism and Developmental Disorders,9(2), 153-159.

Hofer, S., \& Frahm, J. (2006). Topography of the human corpus callosum revisited-comprehensive fiber tractography using diffusion tensor magnetic resonance imaging. Neuroimage,32(3), 989-994.

Hopkins, W. D., \& Rilling, J. K. (2000). A comparative MRI study of the relationship between neuroanatomical asymmetry and interhemispheric connectivity in primates: Implication for the evolution of functional asymmetries. Behavioral Neuroscience, 114(4), 739-748.

Hynd, G. W., Semrud-Clikeman, M., Lorys, A. R., Novey, E. S., Eliopulos, D., \& Lyytinen, H. (1991). Corpus callosum morphology in attention deficit-hyperactivity disorder: Morphometric analysis of MRI. Journal of Learning Disabilities,24(3), 141-146.

Just, M. A., Cherkassky, V. L., Keller, T. A., Kana, R. K., \& Minshew, N. J. (2007). Functional and anatomical cortical underconnectivity in autism: Evidence from an FMRI study of an executive function task and corpus callosum morphometry. Cerebral Cortex,17(4), 951-961.

Just, M. A., Cherkassky, V. L., Keller, T. A., \& Minshew, N. J. (2004). Cortical activation and synchronization during sentence comprehension in high-functioning autism: Evidence of underconnectivity. Brain,127(Pt 8), 1811-1821.

Keary, C. J., Minshew, N. J., Bansal, R., Goradia, D., Fedorov, S., Keshavan, M. S., et al. (2009). Corpus callosum volume and neurocognition in autism. Journal of Autism and Developmental Disorders,39(6), 834-841.

Leboyer, M., Osherson, D. N., Nosten, M., \& Roubertoux, P. (1988). Is autism associated with anomalous dominance? Journal of Autism and Developmental Disorders, 18(4), 539-551.

Lee, D. J., Chen, Y., \& Schlaug, G. (2003). Corpus callosum: Musician and gender effects. NeuroReport,14(2), 205-209.

Lombardo, M. V., Ashwin, E., Auyeung, B., Chakrabarti, B., Taylor, K., Hackett, G., et al. (2012). Fetal testosterone influences sexually dimorphic gray matter in the human brain. Journal of Neuroscience,32(2), 674-680.

Lord, C., Risi, S., Lambrecht, L., Cook, E. H., Jr, Leventhal, B. L., DiLavore, P. C., et al. (2000). The autism diagnostic observation schedule-generic: A standard measure of social and communication deficits associated with the spectrum of autism. Journal of Autism and Developmental Disorders,30(3), 205-223.

Lord, C., Rutter, M., \& Le Couteur, A. (1994). Autism diagnostic interview-revised: A revised version of a diagnostic interview for caregivers of individuals with possible pervasive developmental disorders. Journal of Autism and Developmental Disorders, 24(5), 659-685.
Luders, E., Cherbuin, N., Thompson, P. M., Gutman, B., Anstey, K. J., Sachdev, P., et al. (2010). When more is less: Associations between corpus callosum size and handedness lateralization. Neuroimage,52(1), 43-49.

Luders, E., Narr, K. L., Zaidel, E., Thompson, P. M., Jancke, L., \& Toga, A. W. (2006). Parasagittal asymmetries of the corpus callosum. Cerebral Cortex, 16(3), 346-354.

McCann, B. S. (1981). Hemispheric asymmetries and early infantile autism. Journal of Autism and Developmental Disorders,11(4), 401-411.

McManus, I. C., Murray, B., Doyle, K., \& Baron-Cohen, S. (1992). Handedness in childhood autism shows a dissociation of skill and preference. Cortex,28(3), 373-381.

Medland, S. E., Duffy, D. L., Wright, M. J., Geffen, G. M., \& Martin, N. G. (2006). Handedness in twins: Joint analysis of data from 35 samples. Twin research and human genetics,9(1), 46-53.

Meyer, B. U., Roricht, S., \& Woiciechowsky, C. (1998). Topography of fibers in the human corpus callosum mediating interhemispheric inhibition between the motor cortices. Annals of Neurology,43(3), 360-369.

Muller, R. A., Behen, M. E., Rothermel, R. D., Chugani, D. C., Muzik, O., Mangner, T. J., et al. (1999). Brain mapping of language and auditory perception in high-functioning autistic adults: A PET study. Journal of Autism and Developmental Disorders, 29(1), 19-31.

Ohnishi, T., Matsuda, H., Hashimoto, T., Kunihiro, T., Nishikawa, M., Uema, T., et al. (2000). Abnormal regional cerebral blood flow in childhood autism. Brain,123(Pt 9), 1838-1844.

Oldfield, R. C. (1971). The assessment and analysis of handedness: The Edinburgh inventory. Neuropsychologia,9(1), 97-113.

Ozonoff, S., Cook, I., Coon, H., Dawson, G., Joseph, R. M., Klin, A., et al. (2004). Performance on Cambridge Neuropsychological Test Automated Battery subtests sensitive to frontal lobe function in people with autistic disorder: Evidence from the collaborative programs of excellence in autism network. Journal of Autism and Developmental Disorders,34(2), 139-150.

Papadatou-Pastou, M., Martin, M., Munafo, M. R., \& Jones, G. V. (2008). Sex differences in left-handedness: A meta-analysis of 144 studies. Psychological Bulletin,134(5), 677-699.

Paul, L. K., Brown, W. S., Adolphs, R., Tyszka, J. M., Richards, L. J., Mukherjee, P., et al. (2007). Agenesis of the corpus callosum: Genetic, developmental and functional aspects of connectivity. Nature Reviews Neuroscience,8(4), 287-299.

Perenin, M. T., \& Vighetto, A. (1988). Optic ataxia: A specific disruption in visuomotor mechanisms. I. Different aspects of the deficit in reaching for objects. Brain, 111(Pt 3), 643-674.

Preis, J. (2006). The effect of picture communication symbols on the verbal comprehension of commands by young children with autism. Focus on Autism and Other Developmental Disabilities, 21(4), 194-208.

Prior, M. R., \& Bradshaw, J. L. (1979). Hemisphere functioning in autistic children. Cortex, 15(1), 73-81.

Puig-Antich, J., Lukens, E., \& Brent, D. (1987). psychosocial schedule for school age children 6-16. Pittsburgh: Western Psychiatric Institute and Clinic.

Rakic, P., \& Yakovlev, P. I. (1968). Development of the corpus callosum and cavum septi in man. Journal of Comparative Neurology, 132(1), 45-72.

Rinehart, N. J., Bradshaw, J. L., Moss, S. A., Brereton, A. V., \& Tonge, B. J. (2001). A deficit in shifting attention present in high-functioning autism but not Asperger's disorder. Autism, 5(1), 67-80.

Robbins, T. W., James, M., Owen, A. M., Sahakian, B. J., Lawrence, A. D., McInnes, L., et al. (1998). A study of performance on tests from the CANTAB battery sensitive to frontal lobe dysfunction in a large sample of normal volunteers: Implications for theories 
of executive functioning and cognitive aging. Cambridge Neuropsychological Test Automated Battery. Journal of the International Neuropsychological Society,4(5), 474-490.

Rojas, D. C., Bawn, S. D., Benkers, T. L., Reite, M. L., \& Rogers, S. J. (2002). Smaller left hemisphere planum temporale in adults with autistic disorder. Neuroscience Letters, 328(3), 237-240.

Rojas, D. C., Camou, S. L., Reite, M. L., \& Rogers, S. J. (2005). Planum temporale volume in children and adolescents with autism. Journal of Autism and Developmental Disorders,35(4), 479-486.

Rojas, D. C., Smith, J. A., Benkers, T. L., Camou, S. L., Reite, M. L., \& Rogers, S. J. (2004). Hippocampus and amygdala volumes in parents of children with autistic disorder. American Journal of Psychiatry, 161(11), 2038-2044.

Ronald, A., Happe, F., Bolton, P., Butcher, L. M., Price, T. S., Wheelwright, S., et al. (2006). Genetic heterogeneity between the three components of the autism spectrum: A twin study. Journal of the American Academy of Child and Adolescent Psychiatry,45(6), 691-699.

Sanchez, M. M., Hearn, E. F., Do, D., Rilling, J. K., \& Herndon, J. G. (1998). Differential rearing affects corpus callosum size and cognitive function of rhesus monkeys. Brain Research,812(1-2), 38-49.

Saron, C. D., Foxe, J. J., Simpson, G. V., \& Vaughan, H. G. J. (2003). Interhemispheric visuomotor activation: Spatiotemporal electrophysiology related to reaction time (the parallel brain). Cambridge, MA: MIT Press.

Scamvougeras, A., Kigar, D. L., Jones, D., Weinberger, D. R., \& Witelson, S. F. (2003). Size of the human corpus callosum is genetically determined: An MRI study in mono and dizygotic twins. Neuroscience Letters,338(2), 91-94.

Schlaug, G., Forgeard, M., Zhu, L., Norton, A., \& Winner, E. (2009). Training-induced neuroplasticity in young children. Annals of the New York Academy of Sciences, 1169, 205-208.

Schlaug, G., Jancke, L., Huang, Y., \& Steinmetz, H. (1995). In vivo evidence of structural brain asymmetry in musicians. Science, 267(5198), 699-701.

Schultz, R. T. (2005). Developmental deficits in social perception in autism: The role of the amygdala and fusiform face area. International Journal of Developmental Neuroscience,23(2-3), $125-141$.

Shallice, T. (1982). Specific impairments of planning. Philosophical Transactions of the Royal Society of London. Series B, Biological sciences, 298(1089), 199-209.

Siegel, B. V., Jr, Asarnow, R., Tanguay, P., Call, J. D., Abel, L., Ho, A., et al. (1992). Regional cerebral glucose metabolism and attention in adults with a history of childhood autism. Journal of Neuropsychiatry and Clinical Neurosciences,4(4), 406-414.

Soper, H. V., Satz, P., Orsini, D. L., Henry, R. R., Zvi, J. C., \& Schulman, M. (1986). Handedness patterns in autism suggest subtypes. Journal of Autism and Developmental Disorders, 16(2), 155-167.

Spencer, M. D., Holt, R. J., Chura, L. R., Suckling, J., Calder, A. J., Bullmore, E. T., et al. (2011). A novel functional brain imaging endophenotype of autism: The neural response to facial expression of emotion. Translational Psychiatry, 1, e19.

Steffenburg, S., Gillberg, C., Hellgren, L., Andersson, L., Gillberg, I. C., Jakobsson, G., et al. (1989). A twin study of autism in Denmark, Finland, Iceland, Norway and Sweden. Journal of Child Psychology and Psychiatry,30(3), 405-416.

Steinmetz, H., Staiger, J. F., Schlaug, G., Huang, Y., \& Jancke, L. (1995). Corpus callosum and brain volume in women and men. NeuroReport,6(7), 1002-1004.

Sullivan, P. F., Daly, M. J., \& O'Donovan, M. (2012). Genetic architectures of psychiatric disorders: The emerging picture and its implications. Nature Reviews Genetics, 13, 537-551.

Tan, U. (1991). Serum testosterone levels in male and female subjects with standard and anomalous dominance. International Journal of Neuroscience, 58(3-4), 211-214.

Tepest, R., Jacobi, E., Gawronski, A., Krug, B., Moller-Hartmann, W., Lehnhardt, F. G., et al. (2010). Corpus callosum size in adults with high-functioning autism and the relevance of gender. Psychiatry Research,183(1), 38-43.

Tomasch, J. (1954). Size, distribution, and number of fibres in the human corpus callosum. Anatomical Record,119(1), 119-135.

Tsai, L. Y. (1982). Brief report: Handedness in autistic children and their families. Journal of Autism and Developmental Disorders, 12(4), 421-423.

Tsai, L. Y. (1983). The relationship of handedness to the cognitive, language, and visuo-spatial skills of autistic patients. British Journal of Psychiatry, 142, 156-162.

Vuoksimaa, E., Eriksson, C. J., Pulkkinen, L., Rose, R. J., \& Kaprio, J. (2010). Decreased prevalence of left-handedness among females with male co-twins: Evidence suggesting prenatal testosterone transfer in humans? Psychoneuroendocrinology, 35(10), 1462-1472.

Wechsler, D. (1999). Wechsler abbreviated scale of intelligence. London: The Psychological Corporation.

Witelson, S. F. (1985). The brain connection: The corpus callosum is larger in left-handers. Science,229(4714), 665-668.

Witelson, S. F. (1987). Neurobiological aspects of language in children. Child Development,58(3), 653-688.

Witelson, S. F. (1989). Hand and sex differences in the isthmus and genu of the human corpus callosum. A postmortem morphological study. Brain,112(Pt 3), 799-835.

Witelson, S. F., \& Goldsmith, C. H. (1991). The relationship of hand preference to anatomy of the corpus callosum in men. Brain Research,545(1-2), 175-182. 